\title{
A!
}

This is an electronic reprint of the original article.

This reprint may differ from the original in pagination and typographic detail.

Far, Mehrnaz Farzam; Martin, Floran; Belahcen, Anouar; Rasilo, Paavo; Awan, Hafiz Asad Ali

\section{Real-Time Control of an IPMSM Using Model Order Reduction}

Published in:

IEEE Transactions on Industrial Electronics

DOI:

10.1109/TIE.2020.2973901

Published: 01/03/2021

Document Version

Peer reviewed version

Please cite the original version:

Far, M. F., Martin, F., Belahcen, A., Rasilo, P., \& Awan, H. A. A. (2021). Real-Time Control of an IPMSM Using Model Order Reduction. IEEE Transactions on Industrial Electronics, 68(3), 2005-2014. [9007620].

https://doi.org/10.1109/TIE.2020.2973901

This material is protected by copyright and other intellectual property rights, and duplication or sale of all or part of any of the repository collections is not permitted, except that material may be duplicated by you for your research use or educational purposes in electronic or print form. You must obtain permission for any other use. Electronic or print copies may not be offered, whether for sale or otherwise to anyone who is not an authorised user. 
(C) 2021 IEEE. This is the author's version of an article that has been published by IEEE. Personal use of this material is permitted. Permission from IEEE must be obtained for all other uses, in any current or future media, including reprinting/republishing this material for advertising or promotional purposes, creating new collective works, for resale or redistribution to servers or lists, or reuse of any copyrighted component of this work in other works. 


\title{
Real-Time Control of an IPMSM Using Model Order Reduction
}

\author{
Mehrnaz Farzam Far, Floran Martin, Anouar Belahcen, Senior Member, IEEE, \\ Paavo Rasilo, and Hafiz Asad Ali Awan
}

\begin{abstract}
In the control of electric drives, inaccurate estimation of the motor parameters affects the robustness of the control. This effect is observed particularly when the linear lumped parameter-based models are employed to represent a machine composed of nonlinear magnetic materials. Finite element (FE) models consider the material nonlinearity accurately. However, implementing these models in a real-time system is challenging due to the computational burden. In this paper, we propose a fastdynamic model, based on a model order reduction method, to control a permanent magnet synchronous machine in a wide range of speed. The stator currents in the rotor frame of reference are given as inputs to a reduced FE model, which computes the nodal values of the magnetic vector potential and thereafter the flux linkages. A discrete-time model is used to control the rotor speed and the stator current components. Experiments on a $2.2 \mathrm{~kW}$ interior permanent magnet synchronous machine verify the viability of the proposed model.
\end{abstract}

Index Terms-Electrical machine, interior permanent magnet, model order reduction, orthogonal interpolation method, real-time control, rotor frame of reference, singular value decomposition.

\section{INTRODUCTION}

$\mathrm{A}$ DVANCES in rare-earth magnets have played an important role in the development of permanent magnet (PM) synchronous machines (PMSMs), in which PM materials substitute the rotor windings to generate the air gap magnetic field. In the last decades, PMSM have received attention from different sectors such as the electric vehicle industry, due to the high torque density, low losses, and high efficiency of these machines [1] - [4]. Traditionally, the vector control methods are applied to smoothly run the machine over a wide range of speeds. In the vector control methods, the stator current is transformed into the rotor frame of reference [5] - [8], resulting in two current components: one along the direct- and the other along the quadrature-axis. This transformation decouples the

This work was supported in part by the Academy of Finland and the Estonian Research Council under grant PUT1260

M. Farzam Far is with the department of Electrical Engineering and Automation, Alto University, Espoo, Finland; and VTT Technical Research Centre of Finland, Espoo, Finland.

F. Martin, is with the Department of Electrical Engineering and Automation, Aalto University, Espoo, Finland.

A. Belahcen is with the Department of Electrical Engineering and Automation, Aalto University, Espoo, Finland; and the Department of flux and torque control of the motor and allows proportionalintegral (PI) controllers to be applied to control the torque, which makes it possible to run the machine at a wide range of speeds by applying the flux-weakening methodology.

When designing a control scheme for a motor assuming magnetically linear core materials, the magnetizing inductances in the rotor frame of reference are constant for different values of the stator current. Nevertheless, in a real machine with a nonlinear magnetic circuit, the magnetic saturation and the magnetic cross coupling cannot be neglected, particularly when the machine is operating in the high-speed flux-weakening region. In this case, the inductances and the magnetic flux components are functions of the current components and neglecting the magnetic saturation and cross coupling in the control design can deteriorate the performance of the system. Fig. 1 is an example of the dependency of the stator flux linkages on both components of the stator current. These results are obtained from a finite element (FE) model of the PMSM under study. Although this machine does not experience high saturation at high currents due to its structural properties, the cross-coupling phenomenon is more vivid on the $q$ component of the flux linkage than on the $d$ component.

Several researchers attempted to obtain a robust control system for motor drives by including the magnetic cross
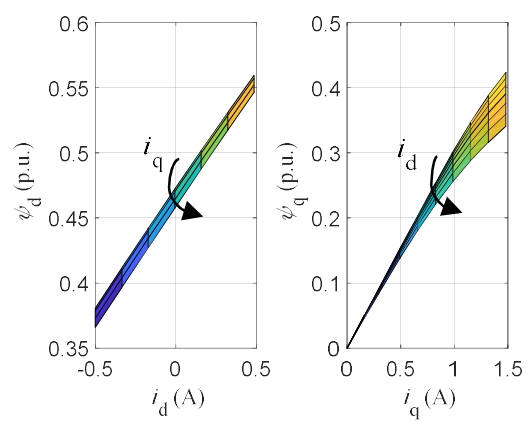

Fig. 1. Computed variation of the flux linkages with respect to the stator current components, i.e. $\psi_{\mathrm{d}}=\psi_{\mathrm{d}}\left(i_{\mathrm{d}}, i_{\mathrm{q}}\right)$ and $\psi_{\mathrm{q}}=\psi_{\mathrm{q}}\left(i_{\mathrm{d}}, i_{\mathrm{q}}\right)$. The arrow show the direction of increasing currents.

Electrical Power Engineering and Mechatronics, Tallinn University of Technology, Estonia.

P. Rasilo, is with the Unit of Electrical Engineering, Tampere University, Tampere, Finland, and with the Department of Electrical Engineering and Automation, Aalto University.

H. A. A. Awan is with ABB Oy Drives, Helsinki, Finland.

Corresponding author e-mail: mehrnaz.farzamfar@vtt.fi. 
coupling and saturation in the model [5], [9] - [11]. The authors of [5] suggested a flux-observer based control scheme for this purpose. However, according to the authors, this scheme is not applicable when using the stationary frame as the reference. Reference [9] proposed a saturated model by defining a single factor saturation as the ratio between the saturated inductance and the unsaturated one. In [10], a nonlinear state equation is applied to include both the iron loss and the magnetic crosssaturation in the vector control of a synchronous reluctance motor. The nonlinear state equation is obtained from an approximate equation, by measuring the inductances in $(d, q)$ coordinates and the iron loss resistance from standstill and noload rotating tests, respectively. The authors of [11] introduced explicit power functions to include the cross-saturation in a motion-sensorless synchronous reluctance machine.

Reference [12] attempted to reduce the effect of parametric sensitivity based on an analytical approach by using look-up tables. Some authors suggested usage of parameter estimation through optimal look-up tables [13] - [18]. In all these methods, errors in the identified or estimated magnetic parameters can lead to the degraded performance. Furthermore, the look-up tables in most of these techniques can be computationally demanding and upsurge the complexity itself. The FE method can solve the electromagnetic system of equations accurately accounting for accurate magnetic circuit geometry and the nonlinear behavior of the material [19]. Nevertheless, a direct implementation of the FE model in a real-time control system is impossible due to the limitation on the computational time. Reference [20] combines a field-circuit-coupled parameter adaptive model with a FE model to consider the crosssaturation, current harmonics, eddy current losses, hysteresis loss, and the influence of temperature on some motor parameters. Although the simulation and experimental results of the proposed method are promising, the implementation of the FE model requires access to a specific black box software to execute real-time simulation.

We use a model order reduction (MOR) technique on the FE model of an electrical machine to derive a faster and accurate reduced model of the machine. The reduced model is then used to control an interior permanent magnet synchronous motor (IPMSM). The dimension and the complexity of this model is significantly lower than that of the FE model and therefore can be used in a real-time application. It should be noted that although the proposed reduced model considers the magnetic saturation and cross-coupling phenomena, the main novelty and advantage of the proposed method is that it can be implemented directly in a real-time application. This paper is an extension to our previous work [21]. To the best knowledge of authors, in the existing literatures, no other MOR techniques have been executed within real-time applications. A dynamic model of an IPMSM and a magnetic model based on MOR are described briefly in Section II. Section III presents the proposed control scheme and the structure of different control blocks. The computation of the current references is based on MOR, resulting in a robust control system against errors created by the magnetic saturation and cross coupling. The proposed control scheme is validated by means of experiments on a $2.2 \mathrm{~kW}$
IPMSM in Section IV. Section V provides the conclusion.

\section{DYNAMIC MODEL OF IPMSM}

\section{A. Fundamental equations}

Considering the equivalent circuit of an IPMSM [18], one can write the stator voltage equation in $(d, q)$ rotor coordinates as

$$
\mathbf{u}_{\mathrm{dq}}=R_{\mathrm{s}} \mathbf{i}_{\mathrm{dq}}+\omega_{\mathrm{r}} \mathbf{J}_{\mathrm{dq}}+\frac{d \boldsymbol{\psi}_{\mathrm{dq}}}{d t}, \quad \mathbf{J}=\left[\begin{array}{cc}
0 & -1 \\
1 & 0
\end{array}\right]
$$

where $\boldsymbol{\psi}_{\mathrm{dq}}, \mathbf{u}_{\mathrm{dq}}$, and $\mathbf{i}_{\mathrm{dq}}$ are vectors containing the $d$ - and $q$-axes components of the flux linkage, stator voltage, and stator current, respectively. $R_{\mathrm{s}}$ is the stator resistance. $\omega_{\mathrm{r}}$ is the rotor electrical angular speed and equals to the time derivative of the rotor angular position: $\omega_{\mathrm{r}}=d \theta_{\mathrm{r}} / d t$. $\mathbf{J}$ is the orthogonal rotation matrix. In linear magnetic materials, the flux linkages are:

$$
\begin{gathered}
\boldsymbol{\psi}_{\mathrm{dq}}=\mathbf{L i}_{\mathrm{dq}}+\boldsymbol{\psi}_{\mathrm{pm}}, \\
\mathbf{L}=\left[\begin{array}{cc}
L_{\mathrm{d}} & 0 \\
0 & L_{\mathrm{q}}
\end{array}\right], \quad \boldsymbol{\psi}_{\mathrm{pm}}=\left[\begin{array}{c}
\psi_{\mathrm{pm}} \\
0
\end{array}\right],
\end{gathered}
$$

where $L_{\mathrm{d}}$ is the $d$-axis inductance, $L_{\mathrm{q}}$ is $q$-axis the inductance, and $\psi_{\mathrm{pm}}$ is the PM flux. In this case, the inductances have constant values with no cross coupling between the axes.

The electromagnetic torque $T$ is computed from the flux linkages, currents, and the number of pole pairs $p$ :

$$
T=\frac{3}{2} p\left(\psi_{\mathrm{d}} i_{\mathrm{q}}-\psi_{\mathrm{q}} i_{\mathrm{d}}\right) .
$$

The generated electromagnetic torque consists of two components: the field torque and the reluctance torque [22].

\section{B. Magnetic model based on MOR}

As mentioned previously, the magnetic circuit of an actual machine experiences saturation as the magnetic flux increases. In this section, we propose a reduced-order FE model, which takes into account the cross-saturation characteristic of the motor and can be applied in real-time control. The FE method is an efficient tool in solving boundary-value problems. In our case, the problem under study is an electrical machine with a magnetostatic field. The solution of this field is searched in terms of a magnetic vector potential A. Having the potential, one can compute the distribution of the flux density $\mathbf{B}=\nabla \times \mathbf{A}$ and field strength $\mathbf{H}=v \mathbf{B}$, where $v$ is the magnetic reluctivity of the material, as well as other quantities such as flux linkages of the windings. The flux linkages obtained in this way consider the magnetic saturation characteristic of the motor and can therefore be applied in a control system to compute the current components from the torque. In the following, we first present the fundamental equations of the FE model and the proposed reduced model. Thereafter, we provide the required equations for evaluating the flux linkages from the magnetic vector potential.

The magnetostatic field in the cross-section of a PMSM is described by Ampere's law as

$$
\nabla \times(v \nabla \times \mathbf{A})=\mathbf{J}+\nabla \times \mathbf{H}_{\mathrm{c}}
$$

where $\mathbf{J}$ is the current density distribution, nonzero only in the current-carrying coils. $\mathbf{H}_{\mathrm{c}}$ is the coercive field strength of the 
PM. The discretized weak form of the field problem can be presented as an algebraic system of equations by using FE method:

\section{Sa}

(5) where $\mathbf{S}$ is the stiffness matrix with size of $n \times n$, with $n$ to be the total number of nodes in the FE mesh. $\mathbf{a}=\left[a_{1} \ldots a_{n}\right]^{\mathrm{T}}$ is the vector of the nodal values of the vector potential, and $\mathbf{f}$ is $n$ source vector resulting from $\mathbf{J}$ and $\nabla \times \mathbf{H}_{\mathrm{c}}$. In nonlinear materials, the stiffness matrix depends on a and a NewtonRaphson iteration scheme is often applied in solving (5).

Various MOR techniques are available to reduce the order of (5). In reference [23], we introduced an efficient MOR technique called the orthogonal interpolation method (OIM) and demonstrated its high computational efficiency in terms of accuracy and computation time, compared to the FE method and another MOR method.

Here, we propose to use the OIM to calculate the nodal values of the magnetic vector potential, and consequently the flux linkage components of the stator. Reference [23] considers only one variable, the amplitude of the stator current, as the input of the reduced model, whereas here we have two variables, the stator current components in the rotor coordinate system, i.e. $i_{\mathrm{d}}$ and $i_{\mathrm{q}}$.

The first step in constructing the OIM model is to solve the FE system of equation at $k \cdot m$ different operating points of $i_{\mathrm{d}}=$ $i_{\mathrm{d} 1} \ldots i_{\mathrm{d} k}$ and $i_{\mathrm{q}}=i_{\mathrm{q} 1} \ldots i_{\mathrm{q} m}$. The corresponding solutions, nodal values of the magnetic vector potential in our case, are stored in an $n \times(k \cdot m)$ matrix $\mathbf{A}_{\mathrm{s}}$. This matrix is known as snapshot matrix and is factorized via a singular value decomposition (SVD) into three matrices as $\mathbf{A}_{\mathrm{s}}=\mathbf{U} \Sigma \mathbf{V}^{\mathrm{T}}$, where ${ }_{\Sigma}$, with the same size as $\mathbf{A}_{\mathrm{s}}$, is a diagonal matrix containing the singular values of $\mathbf{A}_{\mathrm{s}}$. The sum of the square of the singular values defines the total energy of the system [24]. The matrix $\mathbf{U}$ with size of $n \times n$ consists of orthonormal columns that are the eigen modes of the system and the orthonormal columns of $\mathbf{V}$ define how these modes respond to different current inputs. While the product of the matrices $\mathbf{U}$ and $\Sigma$ is constant for any arbitrary input variables within the entire domain of $\mathbf{A}_{\mathrm{s}}$, each column of $\mathbf{V}^{\mathrm{T}}$ corresponds to a unique pair of input $i_{\mathrm{d}}$ and $i_{\mathrm{q}}$ as explained in [23]. The above-described procedure is sketched below:

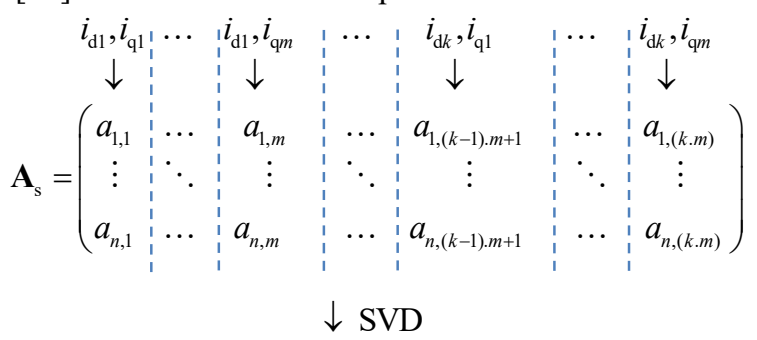

$\mathbf{V}^{\mathrm{T}}=\left(\begin{array}{c:c:c:c:c:c:c}v_{1,1} & \cdots & v_{1, m} & \cdots & v_{1,(k-1) \cdot m+1} & \cdots & v_{1,(k \cdot m)} \\ \vdots & \ddots & \vdots & \ddots & \vdots & \ddots & \vdots \\ v_{(k, m), 1} & \cdots & v_{(k, m), m} & \cdots & v_{(k, m),(k-1) \cdot m+1} & \cdots & v_{(k, m),(k . m)}\end{array}\right)$.

Each row of $\mathbf{V}^{\mathrm{T}}$ can be presented as a function associated with the inputs $i_{\mathrm{d}}$ and $i_{\mathrm{q}}$. These functions are predefined in the OIM block and one can directly compute the new components of $\mathbf{V}_{\text {new }}^{\mathrm{T}}$ for any new values of $i_{\mathrm{d}}$ and $i_{\mathrm{q}}$ during the real time control of the machine. The corresponding nodal values of the magnetic vector potential are obtained as

$$
\mathbf{a}_{\text {new }}=\mathbf{U} \Sigma \mathbf{V}_{\text {new }}^{\mathrm{T}} .
$$

One of the key features of OIM, which makes it compatible with the real-time application, is the ability of the method in actual order reduction of the problem. $\mathbf{V}^{\mathrm{T}}$ is a square matrix of size $(k \cdot m)$. However, according to the fundamental principle of SVD, the row space of $\mathbf{A}_{\mathrm{s}}$ is spanned into the first $r$ rows of $\mathbf{V}^{\mathrm{T}}$ by capturing the mode with the highest energy and the null space of $\mathbf{A}_{\mathrm{s}}$ is spanned by the remaining $(k \cdot m)-r$ rows of $\mathbf{V}^{\mathrm{T}}$ [25]. As a result, $r(r<<(k \cdot m))$ functions are sufficient to accurately represent the vector $\mathbf{V}_{\text {new }}^{\mathrm{T}}$. In the same manner, the sizes of $\mathbf{U}$ and $\Sigma$ are reduced to $\mathbf{U}_{\mathrm{r}}$ with size of $n \times(k . m-r)$ and $\Sigma_{\mathrm{r}}$ with size of $(k \cdot m-r) \times(k \cdot m-r)$, respectively. The value of $r$ equals to the number of singular values that can capture most of the energy of the system [24].

Now that $\mathbf{a}_{\text {new }}$ is known, the next step is to express the stator flux linkage in terms of the nodal values of the magnetic vector potential. The flux linkage for phase $x$ of a 3 -phase winding, in terms of the nodal values of the magnetic vector potential, is in the form of

$$
\psi_{x, \text { new }}=\sum_{j=1}^{n} C_{x, j} a_{j, \text { new }}, \quad x=1, \ldots, 3
$$

in which $a_{j \text {,new }}$ is the new nodal value of the magnetic vector potential for node $j$ and the coefficients $C_{j}$ are defined as

$$
C_{x, j}=\frac{K_{x} l}{S_{\mathrm{T} x}} \int_{\Omega} \delta_{x} N_{j} \mathrm{~d} \Omega,
$$

where $k_{x}$ is the number of turns of the winding $x$ and $S_{\mathrm{T} x}$ is the total cross-sectional area of one side of the winding $x . N_{j}$ is the FE shape function of node $j$. The integral is performed over the coil areas. The value of $\delta_{x}$ depends on the location of the element whether the element is in positive coil sides $\left(\delta_{x}=1\right)$, negative coil side $\left(\delta_{x}=-1\right)$, or elsewhere $\left(\delta_{x}=0\right)$. The flux linkage in the $(d, q)$ rotor coordinate frame is computed by using Park's transformation.

It should be noted that all the terms in (9) are constant and known for a given machine design, which means $C_{j}$ can be computed once and in advance of the flux computation. Moreover, the total flux linkages of the stator phase windings could be computed by knowing the nodal values of the magnetic vector potentials at the coils' cross-sections. This results in further computational reduction, since only few entries of $\mathbf{a}_{\text {new }}$, and consequently $\mathbf{U}_{\mathrm{r}} \Sigma_{\mathrm{r}}$ and $\mathbf{V}_{\text {new }}^{\mathrm{T}}$ are required.

To conclude this section, we derived a reduced model from the FE model of an electrical machine. As it will be shown later, this model can be presented as a black box with the current components of the machine as the inputs and the flux components as the outputs. If the reduced model considers the nonlinearity of the actual electrical machine accurately, the black box can be placed in the control system scheme at places where the relation between current and flux components are required. 


\section{Control System BAsed on the Reduced Model}

Fig. 2 shows the proposed control block diagram for the machine under study. A discrete-time model is used to control the current components of the stator. The $d$-axis current is controlled according to the required stator flux linkage and the $q$-axis current is regulated to produce the required torque [26].

An encoder is employed to determine the rotor position $\theta$ and the actual speed of the machine $\omega_{\mathrm{r}}$. A typical proportionalintegral (PI) speed controller is used to obtain the torque reference $T_{\text {ref. }}$ The reference current components $\mathbf{i}_{\mathrm{dq}, \text { ref }}$ and the corresponding values of inductances $\mathbf{L}_{\mathrm{dq}}$ are calculated by means of the OIM, which is embedded in the current reference block. These values, along with the measured current $\mathbf{i}_{\mathrm{dq}}$, are then passed to the discrete-time current controller to generate the pertinent voltage reference $\mathbf{u}_{\mathrm{dq}, \text { ref. }}$ The current reference, OIM, and current controller blocks are explained thoroughly in the next subsections.

The pulse-width modulator (PWM) computes the duty ratios in response to the voltage reference and the DC-bus voltage $U_{\mathrm{dc}}$. The sampling of $\mathbf{i}_{\mathrm{dq}}$ is synchronized with the PWM. The computational delay in the control system produces an angular error of $\omega_{\mathrm{r}} T_{\mathrm{s}}$, with a sampling period of $T_{\mathrm{s}}$.

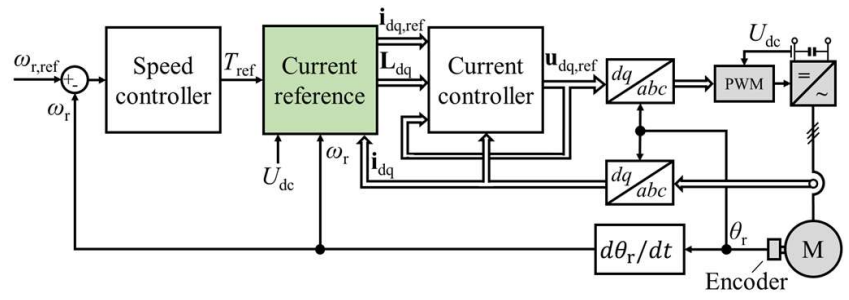

Fig. 2. Block diagram of the control system for an IPMSM drive. The reduced order model is embedded in the current reference block to compute current references and inductance components. Single lines and double lines represent scalar and vector quantities, respectively.

\section{A. Structure of the current reference block}

To consider the magnetic saturation effect and the coupling between the current components, we propose to implement the reduced order model in the current reference block. Fig. 3 presents the internal structure of the current reference block.

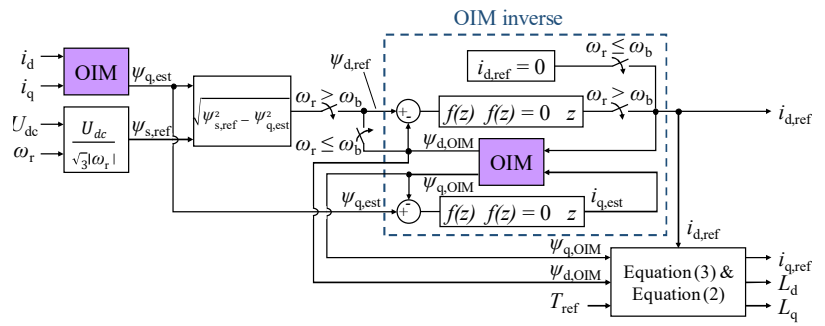

Fig. 3. Internal structure of the current reference block.

This block consists of two main sub-blocks. The first one is an OIM block that computes the actual value of the flux linkage in $q$-axis, $\psi_{\mathrm{q} \text {,est }}$; and the second sub-block is the OIM inverse, which determines the $d$ component of the reference current $i_{\mathrm{d}, \mathrm{ref}}$ as well as the flux linkages $\psi_{\mathrm{d} \text {,est }}$ and $\psi_{\mathrm{q} \text {,est. }}$.

Here, we use an 'algebraic constraint', a Simulink feature, in conjunction with an OIM block to build the OIM inverse. The algebraic constraint block operates in a manner to curb the input $f(z)$ to zero and generates the output $z$ accordingly. To maintain this sequence of operation, it must have a feedback path that keeps the input at zero. The resolution of this non-linear system of equation only needs few steps so that within a few sampling periods, the $d-q$ reference currents are determined to match with the $d-q$ reference fluxes.

The working principle of OIM inverse depends upon the speed of the machine. For speed below the base speed, i.e. the constant torque region, we enforce the $d$-axis current reference $\boldsymbol{i}_{\mathrm{d}, \text { ref }}$ to be zero, compute the flux linkage components via an OIM block and then calculate $i_{\mathrm{q}, \text { ref }}, L_{\mathrm{d}}$, and $L_{\mathrm{q}}$. The $i_{\mathrm{d}}=0$ control principle is suitable for this particular machine under study since it has low inductances and insignificant armature reaction. For machines with high inductances and armature reaction, the maximum torque-per-ampere (MTPA) trajectory [27] is applied to minimize the copper losses while maintaining the maximum torque.

If the speed of the machine is above the base speed, i.e. within the flux-weakening region, the control of the machine is no longer possible through the $i_{\mathrm{d}}=0$ control principle. Instead, a demagnetizing flux is introduced that repeals the fixed flux produced by the PMs. The most feasible means to accomplish this aim is the instigation of a negative $d$ component of current that fosters the opposing flux.

As for the flux-weakening region, the control is designed to acquire the $d$ component of the flux $\psi_{\mathrm{d}}$ according to the fluxweakening conditions and then generate the current component in the same axis that supports the required flux. The reference $d$ component of the flux linkage $\psi_{\mathrm{d} \text {,ref }}$ is obtained from $\psi_{\mathrm{q}, \mathrm{est}}$ and the magnitude of the flux linkage reference $\psi_{\mathrm{s} \text {,ref. }}$ It is to be noted that in this region $\psi_{\mathrm{s} \text {, ref }}$ is computed by maximizing the power per volt, as $U_{\mathrm{dc}} /\left(\sqrt{3} \omega_{\mathrm{r}}\right)$, where $U_{\mathrm{dc}}$ is the voltage limitation of the inverter.

Here, the error between $\psi_{\mathrm{d} \text {,ref }}$ and $\psi_{\mathrm{d} \text {,OIM }}$ behaves as $f(z)$ and $i_{\text {d,ref }}$ relates with $z$ state. To consider the cross coupling phenomena, a secondary algebraic constraint block is used to provide the input $i_{\text {q,est }}$ for the OIM block. The input of this block is the difference between $\psi_{\mathrm{q} \text {,est, }}$ previously obtained from measured $i_{\mathrm{q}}$, and the $q$ component of the flux $\psi_{\mathrm{q} \text {,OIM }}$ from the OIM block.

Having $i_{\mathrm{d} \text {,ref, }} \psi_{\mathrm{d}}$, and $\psi_{\mathrm{q}}$, one can compute the $q$ component of the current reference $i_{\mathrm{q}, \text { ref }}$ and the inductance components $L_{\mathrm{d}}$ and $L_{\mathrm{q}}$ from (3) and (2), respectively. The values of $i_{\mathrm{d}, \text { ref }}$ and $i_{\mathrm{q}, \text { ref }}$ to be passed to the current controller depend also on the current limit. The resultant $\sqrt{i_{\mathrm{d}, \text { ref }}^{2}+i_{\mathrm{q}, \text { ref }}^{2}}$ is restrained from exceeding the maximum current limit of the setup.

\section{CONSTRUCTION OF THE OIM BLOCK}

The OIM is employed to reduce the order of the FE model of a $2.2 \mathrm{~kW}$ six pole IPMSM. The machine is simulated with second order finite element with 1379 nodes and current supply. The rated values and parameters of the motor are provided in Table I (for the geometry and magnetic material properties see [28], [29]). The accuracy of the FE model of the motor was 
TABLE I

PARAMETERS OF THE IPMSM

\begin{tabular}{ll}
\hline \hline Parameter & Value \\
\hline \hline Connection & Delta \\
\hline Power & $2.2 \mathrm{~kW}$ \\
\hline Rated voltage (rms) & $370 \mathrm{~V}$ \\
\hline Rated current (rms) & $4.3 \mathrm{~A}$ \\
\hline Number of pole pairs & 3 \\
\hline Frequency & $75 \mathrm{~Hz}$ \\
\hline Rated speed & $1500 \mathrm{r} / \mathrm{min}$ \\
\hline Torque & $14 \mathrm{Nm}$ \\
\hline Direct-axis inductance & $36 \mathrm{mH}$ \\
\hline Quadrature-axis inductance & $53 \mathrm{mH}$ \\
\hline Stator resistance & $3.59 \Omega$ \\
\hline Permanent magnet flux-linkage & $0.555 \mathrm{Vs}$ \\
\hline Moment of inertia of rotor and load & $0.015 \mathrm{~kg} \cdot \mathrm{m}^{2}$ \\
\hline \hline
\end{tabular}

previously validated by comparing the measured currents with simulated ones [30].

One of the main factors affecting the accuracy of the reduced model is how the range of $i_{\mathrm{d}}$ and $i_{\mathrm{q}}$ are chosen when generating the snapshot matrix. This range must cover all the possible operating points of the machine. Due to the nature of our control method, $i_{\mathrm{d}}$ is expected to vary from negative rated current to zero and $i_{\mathrm{q}}$ changes from negative rated current to positive rated current. Therefore, the FE model is solved for 40 operating points, chosen from all the possible combinations of 5 values of $i_{\mathrm{d}}$, equally distributed in $\left[-I_{\mathrm{N}}, 0\right]$, and 8 values of $i_{\mathrm{q}}$, equally distributed in $\left[-I_{\mathrm{N}}, I_{\mathrm{N}}\right]$, with $I_{\mathrm{N}}$ being the rated current. It should be noted that in some cases during the operation of the motor, higher values of current components might be drawn from machine. Nevertheless, one advantage of the OIM is that it can evaluate the system solution with an acceptable precision for any input values up to about $20 \%$ of the upper and lower bound of the selected range of $i_{\mathrm{d}}$ and $i_{\mathrm{q}}$ [29].

Next, the snapshot matrix is decomposed via SVD. In our case, the first five singular values capture about $95 \%$ of the total energy of the system [24]. This means that the first five rows of $\mathbf{V}^{\mathrm{T}}$, and thus five functions $\left(f_{1}, \ldots, f_{5}\right)$, are sufficient to represent the matrix $\mathbf{V}^{\mathrm{T}}$. These functions are illustrated in Fig. 4. In this particular case, the first three functions represent three angled planes $\mathrm{V}_{1}, \mathrm{~V}_{2}$, and $\mathrm{V}_{3}$; and the last two functions represent two hyperbolic paraboloids $\mathrm{V}_{4}$, and $\mathrm{V}_{5}$.

In the real-time operation, for any given $i_{\mathrm{d}}$ and $i_{\mathrm{q}}$, the entries of $\mathbf{V}_{\text {new }}^{\mathrm{T}}=\left[v_{1}, \ldots, v_{5}\right]^{\mathrm{T}}$ and the flux linkage components are accurately computed by using these functions, (7), and (8). Since the coefficients $C_{x, j}$ in (8) are known prior to the calculation of flux linkages, we compute these coefficients in the $(d, q)$ coordinate system and store them in a matrix $\mathbf{C}$. The IPMSM has 210 nodes at the coils' cross-sections, which results the size of $\mathbf{C}$ to be $2 \times 210$ and reduces the size of $\mathbf{U}_{\mathrm{r}} \Sigma_{\mathrm{r}}$ to 210 $\times 5$, as mentioned previously. For further simplicity, the product of $\mathbf{C}$ and $\mathbf{U}_{\mathrm{r}} \Sigma_{\mathrm{r}}$, which is a $2 \times 5$ matrix, is computed in advance and defined in the OIM block as shown in Fig. 5.
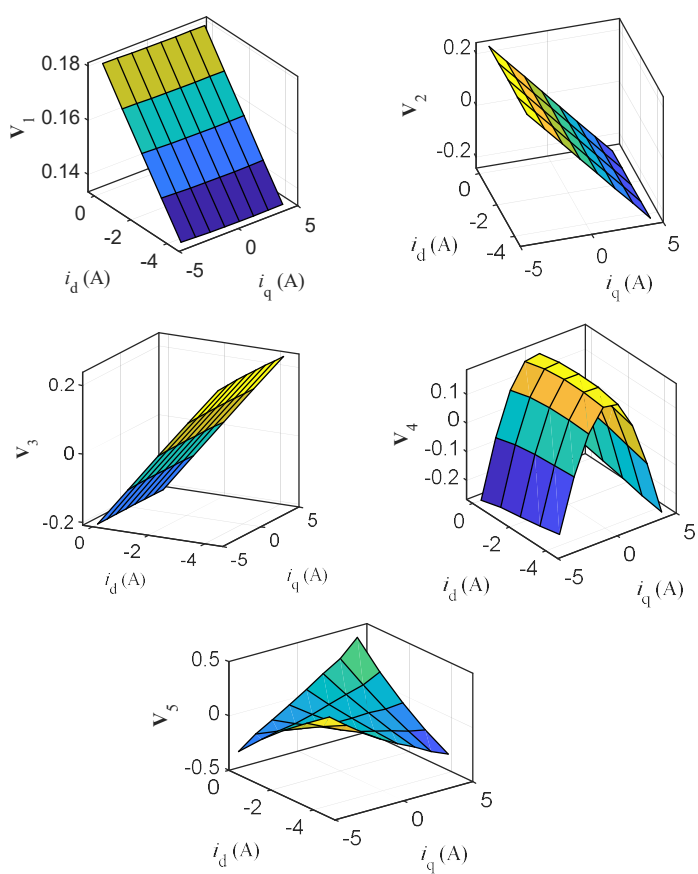

Fig. 4. The first five rows of matrix $\mathbf{V}^{\top}$ are presented as orthogonal functions of the current components $i_{\mathrm{d}}$ and $i_{\mathrm{q}}$.

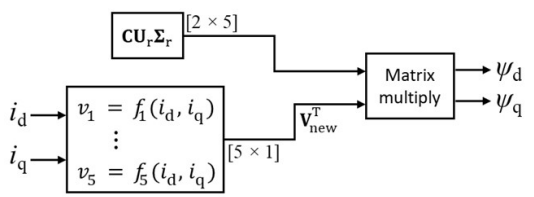

Fig. 5. Internal structure of OIM block.

\section{B. Structure of current controller block}

Reference [31] presents a discrete-time current control design for synchronous motor drives. We adapt a similar discrete-time approach to design the current controller. The control law for a two-degree-of-freedom (2DoF) state-space flux linkage controller with an integral action in the $z$-domain is expressed as

$$
\begin{aligned}
\mathbf{u}_{\mathrm{dq}, \mathrm{ref}}(z)= & \mathbf{K}_{\mathrm{t}} \mathbf{i}_{\mathrm{dq}, \mathrm{ref}}(z)+\frac{\mathbf{K}_{\mathrm{i}}}{z-1}\left[\mathbf{i}_{\mathrm{dq}, \mathrm{ref}}(z)-\mathbf{i}_{\mathrm{dq}}(z)\right] \\
& -\mathbf{K}_{1} \mathbf{i}_{\mathrm{dq}}(z)-\frac{\mathbf{K}_{2}}{z} \mathbf{u}_{\mathrm{dq}, \mathrm{ref}}(z),
\end{aligned}
$$

where $\mathbf{K}_{\mathrm{t}}$ is the feedforward gain, $\mathbf{K}_{\mathrm{i}}$ is the integral gain, $\mathbf{K}_{1}$ and $\mathbf{K}_{2}$ are the state-feedback gains. These gain matrices are defined as

$$
\begin{gathered}
\mathbf{K}_{2}=\mathbf{I}+\mathbf{B}^{-1}(-2 \beta \mathbf{I}+\mathbf{A}) \mathbf{B}, \\
\mathbf{K}_{1}=\mathbf{B}^{-1}\left(\beta^{2} \mathbf{I}-\mathbf{A}\right)+\mathbf{K}_{2} \mathbf{B}^{-1}(\mathbf{I}+\mathbf{A}), \\
\mathbf{K}_{\mathrm{i}}=\mathbf{K}_{1}-\mathbf{K}_{2} \mathbf{B}^{-1} \mathbf{A}, \quad \mathbf{K}_{\mathrm{t}}=\mathbf{B}^{-1}(1-\beta) \mathbf{I},
\end{gathered}
$$

where $\mathbf{I}$ is the identity matrix of size $2, \beta=\exp \left(-\alpha T_{\mathrm{s}}\right)$, and $\alpha$ is the current controller bandwidth. The rotor speed is considered in the gains by the matrices $\mathbf{A}$ and $\mathbf{B}$ :

$$
\begin{gathered}
\mathbf{B}=T_{\mathrm{s}} e^{\left(\omega_{\mathrm{r}} T_{\mathrm{s}} / 2\right) \mathbf{J}}\left(\mathbf{I}+T_{\mathrm{s}}\left(-R_{\mathrm{s}} \mathbf{L}_{\mathrm{OIM}}^{-1}-\omega_{\mathrm{r}} \mathbf{J}\right) / 2\right), \\
\mathbf{A}=\left(\mathbf{I}+T_{\mathrm{s}}\left(-R_{\mathrm{s}} \mathbf{L}_{\mathrm{OIM}}^{-1}-\omega_{\mathrm{r}} \mathbf{J}\right)\right)+T_{\mathrm{s}}^{2}\left(-R_{\mathrm{s}} \mathbf{L}_{\mathrm{OIM}}^{-1}-\omega_{\mathrm{r}} \mathbf{J}\right)^{2},
\end{gathered}
$$


in which $\mathbf{L}_{\mathrm{OIM}}^{-1}$ is the inverse of inductance matrix:

$$
\mathbf{L}_{\mathrm{OIM}}^{-1}=\left[\begin{array}{cc}
1 / \mathrm{L}_{\mathrm{d}} & 0 \\
0 & 1 / \mathrm{L}_{\mathrm{q}}
\end{array}\right] .
$$

In order to account for the cross-saturation, the inductances $L_{\mathrm{d}}$ and $L_{\mathrm{q}}$ are obtained during the real-time operation from the current reference block as explained in Section III A.

It should be noted that the current control loop stability is not affected if the values of inductances obtained from (13) are not far away from the actual inductances. The current controller should be able to handle small changes in the parameters of the motor by integral action of the controller. The effect of change in inductances on the current controller is discussed in detail in the reference [31].

\section{REsults}

The proposed control scheme is implemented in the real time control of the $2.2 \mathrm{~kW}$ IPMSM drive. Fig. 6 provides a picture of the experimental setup. A PM servomotor is coupled with the IPMSM as the load of the drive system. The IPMSM and the servomotor are supplied with a customized Danfoss VLT5004 frequency converter and a commercial Bivector frequency converter, respectively. The proposed control algorithm is implemented in the MATLAB/Simulink environment. The simulated control system is then compiled and employed in a dSPACE DS1103 PPC processor board. The DS1103 is connected to the other components of the system through a signal-conditioning unit (SCU). The inertia of the whole system is $0.015 \mathrm{~kg} \cdot \mathrm{m}^{2}$.

The effects of magnetic saturation and cross coupling on the operation of the control system are studied by comparing the proposed control (PC) method with a constant inductances control (CC) method. Here, the constant inductances control method refers to a control model in which the OIM is replaced by (2) and the rated values of the inductance components are used when required. Fig. 7 shows the maximum torque and output power that are produced by the PC and CC methods. The $\mathrm{PC}$ method provides higher torque and power at most of the given speeds. Particularly within the flux-weakening region, in which the torque and power production is almost double that for the CC method. This superior performance of PC method is understandable considering the magnetizing curve in Fig. 8. In the CC method, the inductance of the device is constant, and a

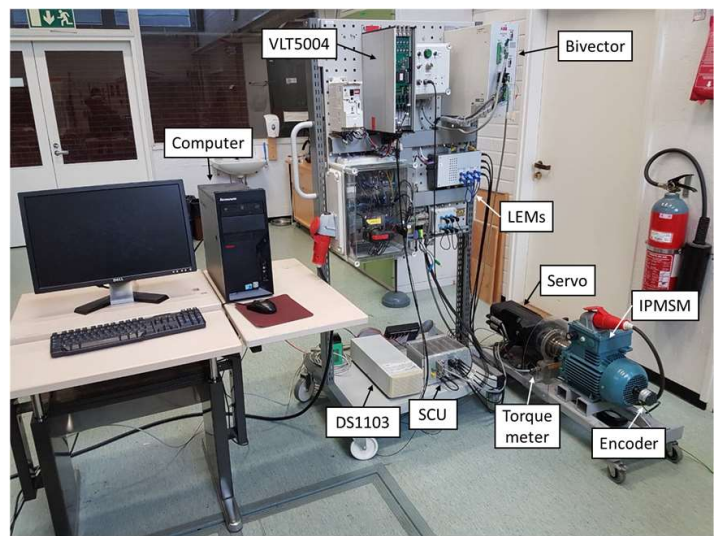

Fig. 6. Picture of the laboratory setup. linearization is assumed when computing the flux linkage from current. However, in the PC method, the inductance, and therefore the flux linkage, saturates as a function of current. As shown in Fig. 8, for any current less than the rated current $I_{\mathrm{N}}$, the PC method produces higher flux linkage. Moreover, due to nonlinearity, the magnetization curve of this method reaches the rated current at a slower rate in comparison with that of the $\mathrm{CC}$ control method.

Fig. 9 shows the magnitude of the measured currents at different torque and speed points. Fig. 10 presents the corresponding current angles only at the flux-weakening region. Although no systematic pattern is recognized between the current magnitudes of the two methods, the proposed method provides higher torque when its current magnitude is higher than that of the $\mathrm{CC}$ method. As for the current angles, the $\mathrm{CC}$ method moves faster towards $180^{\circ}$. This means the this method draws higher $i_{\mathrm{d}}$ in negative direction, which in return results to larger demagnetization of the permanent magnet and lower flux magnitude. Furthermore, the effect of increasing the torque and the current is assessed by calculating the electromagnetic efficiency as:

$$
\eta=\frac{P_{\text {out }}}{P_{\text {out }}+3 R I^{2}},
$$

where $P_{\text {out }}=T \cdot \omega_{\mathrm{r}}$ is the output power, $I$ is the magnitude of the measured current, and $R$ is the resistance of the machine. The value of $\eta$ at the maximum torque versus speed is shown in Fig. 11. It should be noted that the efficiency decreases when the current increases. We can infer that even if the current

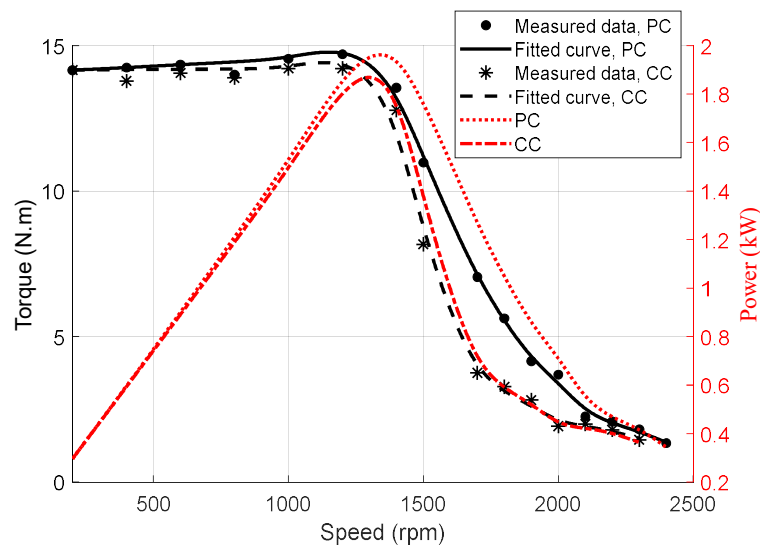

Fig. 7. Maximum torque versus speed characteristics and output power curves for the IPMSM drive, obtained via the PC method and the CC method. Measured values of the torque from these two methods are marked with dots and stars.

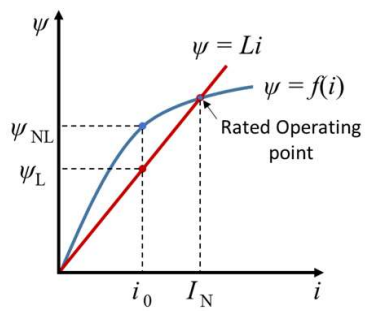

Fig. 8. Magnetizing curve of the conventional (red line) and proposed (blue curve) methods. For any current less than the rated current, the nonlinear curve provides higher flux linkage, as compared with the linear line. 


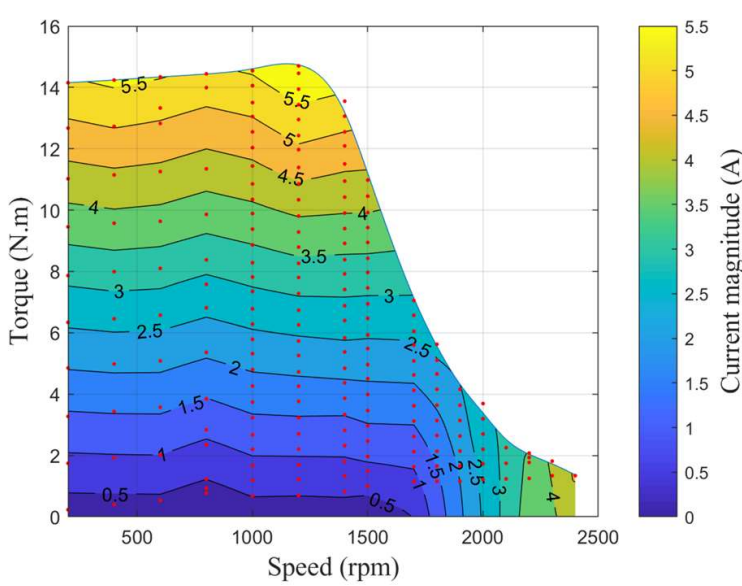

(a)

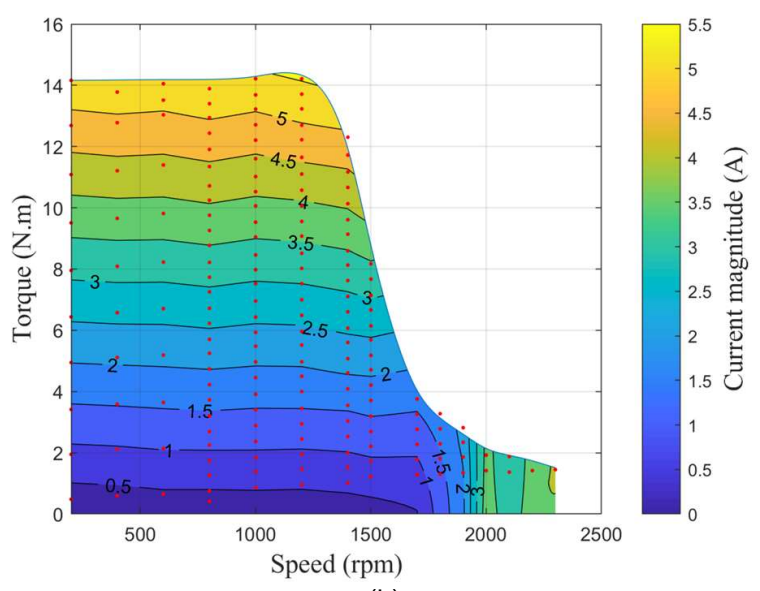

(b)

Fig. 9. Magnitudes of the measured currents of the IPMSM, when the motor is driven by (a) the PC method and (b) the CC method. The red dots mark the torque and the speed at which data were collected.

increases, $\eta$ remains almost the same for both methods.

The performance of the PC method is further investigated experimentally against the performance of a commissioningbased control (COMC) method [32], which also considers the saturation and cross-coupling phenomena. In the commissioning procedure, the computation of the nonlinear flux linkages curves was carried out under a constant rotating speed of $200 \mathrm{rad} / \mathrm{s}$, for an electrical frequency of $31.8 \mathrm{~Hz}$. This frequency was selected low enough in order to diminish the influence of core losses on the flux linkages estimation [33]. The flux linkage reference curves, obtained experimentally via commissioning method, were then introduced as look-up tables in the control system to produce a commissioning-based control system. Fig. 12 compares the torque dynamics of the PC and COMC drives at speed of $1800 \mathrm{rpm}$. The load torque is first changed stepwise from about 0 to $2 \mathrm{Nm}$ and then back to $0 \mathrm{Nm}$. The load torque implementation leads to a slight drop in the speed. However, the speed starts to follow the reference speed after the torque settles down. The speed results are the data collected from the encoder. The values of the actual torque and currents are measured by means of a torque meter and current sensors, respectively. The difference between the load torque and the actual torques comes from the friction torque, which is included in the measured torques. The flux linkage components

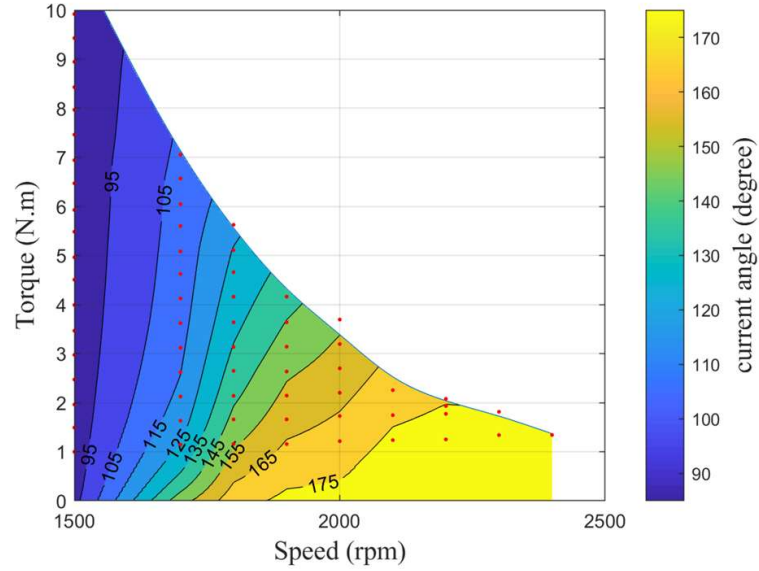

(a)

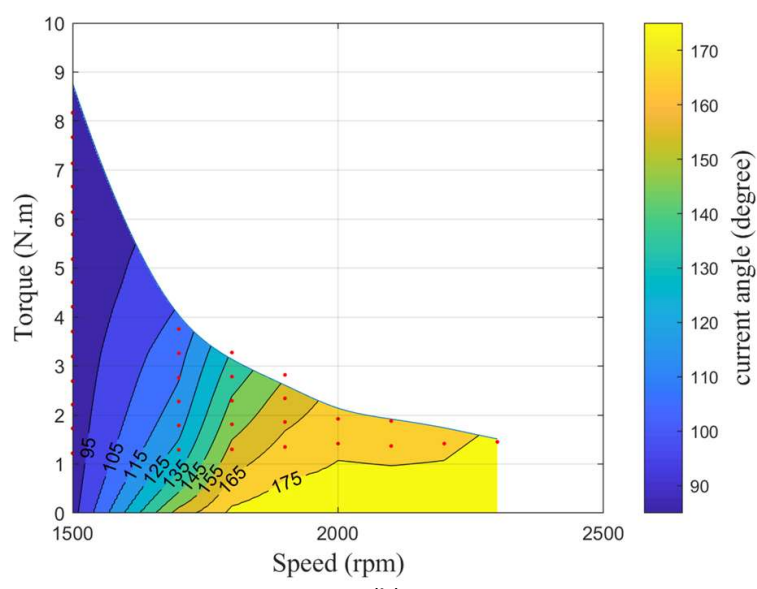

(b)

Fig. 10. Angles of measured currents of the IPMSM, when motor is driven by (a) the PC method and (b) the CC method. The red dots mark the torque and the speed at which data were collected.

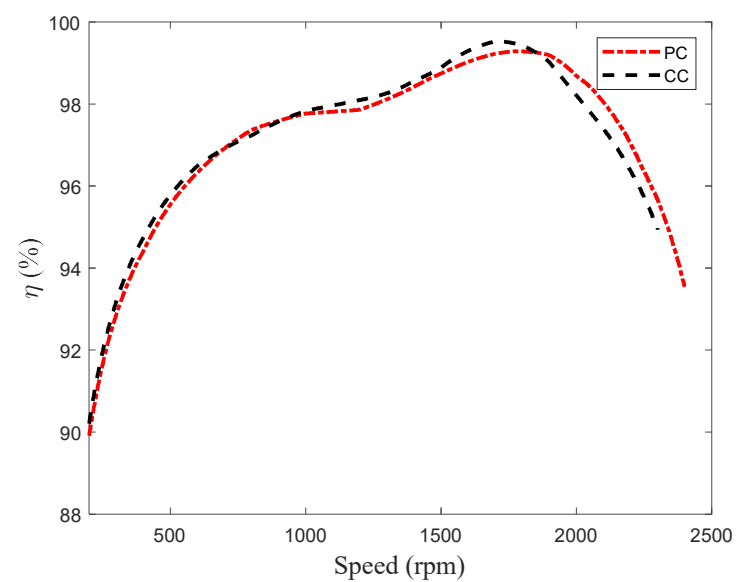

Fig. 11. Electromagnetic efficiency (14) for the PC and CC systems.

are calculated by using the measured currents as input to OIM or the look-up table obtained by commissioning method, depending on the control method. As it can be seen in Fig. 12, both control drives lead to similar torque dynamics. The slight variances in the measured components of the currents in $d$ axis have resulted into minor dissimilarities in the flux linkage components in both $d$ and $q$ axes. 

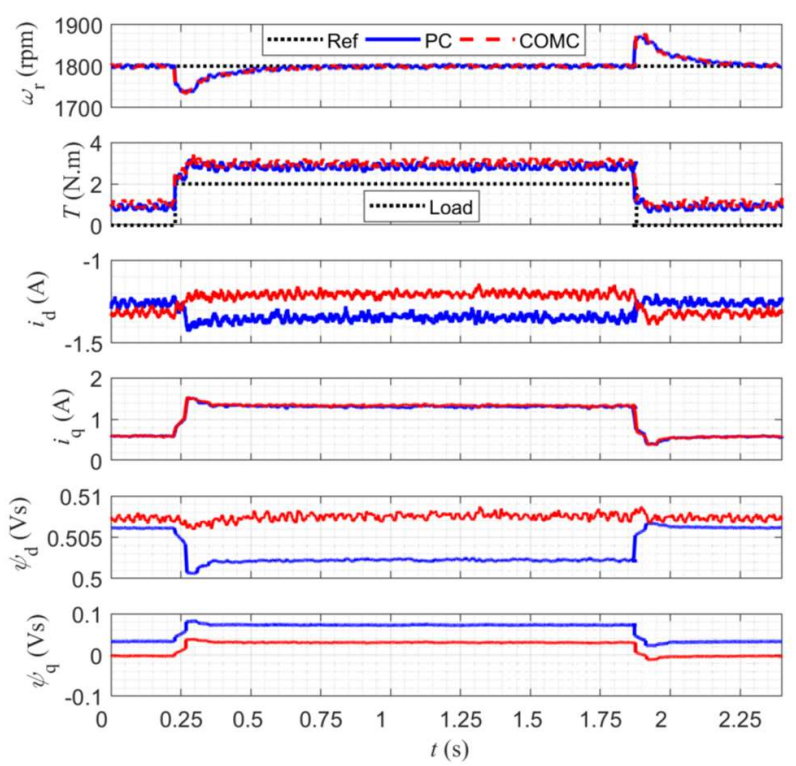

Fig. 12. Experimental results of the $P C$ and $C O M C$ method. The speed reference (Ref) is maintained at $1800 \mathrm{rpm}$ and the load torque (Load) is changed stepwise from 0 to $2 \mathrm{Nm}$ and then back to $0 \mathrm{Nm}$.

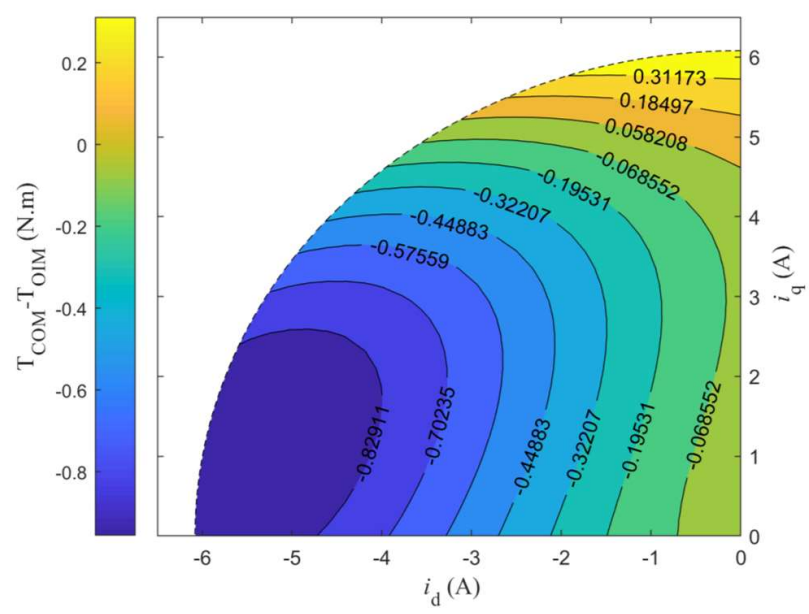

Fig. 13. Difference between the torque values computed by means of the COMC method (Tсом) and PC method (TомM).

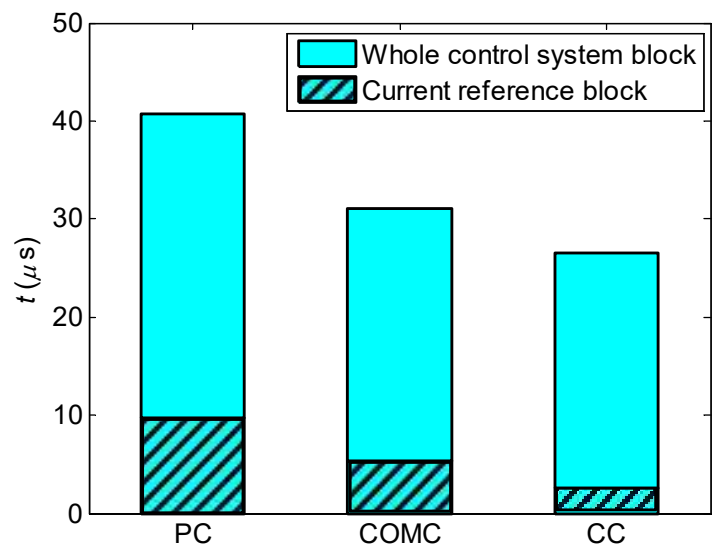

Fig. 14. Comparing the execution time of different control systems.
The COMC method results in higher $i_{\mathrm{d}}$ when torque changes from zero to $2 \mathrm{Nm}$. Nevertheless, the difference between the torque values of COM and PC control methods in $i_{\mathrm{d}}-i_{\mathrm{q}}$ plane, Fig 13, suggests that there are operating points at which the proposed method provides higher torque than the COMC method. Near the base speed, the commissioning method produces higher torque. However, as the speed increases, it underestimates the torque, as compared with the OIM method. This can be explained by the fluctuation of the DC bus voltage while estimating the flux linkage along the $q$ axis during the commissioning procedure [32], [33].

To investigate the computational burden of the PC method, the execution time of this method is compared with the COMC and CC methods. The results of these comparisons are shown in Fig. 14. Each bar shows the required time for running the entire control system over a single simulation step. The hatched area indicates how much of the execution time is consumed by the current reference block. As expected, the execution time of the PC method is slightly higher, since it has a more complicated model and more operations. Nevertheless, during the measurement the computational time did not impose any burden on the real-time computation. The PC method consumes $14 \%$ more CPU than the COMC method and 53\% more CPU than the $\mathrm{CC}$ method. It is worth mentioning that these three control methods have same solver and same sampling period of $200 \mu \mathrm{s}$, but different execution time, due to the differences in their models.

\section{DISCUSSION AND CONCLUSION}

This paper presents the real-time control of an IPMSM drive operating in a wide range of speed via the reduced FE model of the machine. The reduced model is based on OIM, in which the nodal values of the magnetic vector potential are computed for any arbitrary stator winding currents of the machine in the rotor coordinate frame. The $d$ and $q$ components of the flux linkage are then obtained by multiplying the corresponding nodal values of the vector potential with a constant depending of the slot area and the number of turns. The computed flux linkages are accurate and consider the magnetic saturation characteristics. The proposed method is applicable to real-time control of any type of synchronous machine drives. In this paper, however, we use a $2.2 \mathrm{~kW}$ IPMSM coupled with a servomotor as load, to show the feasibility of the method experimentally.

The nonlinear inductance characteristics obtained from the proposed reduced method can be similar to the ones evaluated with industrial methods, such as machine commissioning method. Consequently, it is expected that the control systems based on the reduced method and commissioning methods lead to similar control results as well. This paper shows that the COMC method leads to a better torque-per-amp (TPA) characteristics than the PC method at some operating points, specifically at speed near the base speed. Nevertheless, the PC method provides higher torque at higher speeds, since the COMC method underestimates the torque in the fluxweakening region. This can be explained by the fluctuation of the DC bus voltage while estimating the flux linkage along the 
$q$ axis during the commissioning procedure.

As a limitation on the PC method, it should be pointed out that building the reduced $\mathrm{FE}$ model requires either the knowledge of the dimension or the input-output relation of the machine under investigation. Therefore, for example, the PC model might not be suitable for plug-and-play frequency converters if either of this information is not known. Nevertheless, it can be applied in integrated drives-machine packages, where both the drive and machine specifications are provided together. Since the PC model accounts for the magnetic saturation and cross coupling, it is an adequate substitution for the conventional linear lumped parameterbased control model, especially within the flux-weakening region, in which the variations of the motor parameters are more sensitive to the non-linear behavior of the materials.

The conventional methods that use nonlinear saturation characteristics obtained from commissioning do not consider the core losses when evaluating the optimal references, instead separate look-up tables are required for that. In the case of PC method, the core losses can be included in the reduced model; however, this falls beyond the scope of this paper.

To summarize, the advantages and limitations of the PC method compare to industrial control method are listed in Table II:

TABLE II

ADVANTAGES AND LIMITATIONS OF PC METHOD AS COMPARED WITH COMC METHOD

\begin{tabular}{ll}
\hline \hline Advantages & Limitations \\
\hline \hline $\begin{array}{l}\text { Higher TPA in flux } \\
\text { weakening region, as the } \\
\text { speed limit reached }\end{array}$ & $\begin{array}{l}14 \% \text { higher computational } \\
\text { load }\end{array}$ \\
$-\quad \begin{array}{l}\text { Does not require } \\
\text { additional look-up table } \\
\text { for considering the core } \\
\text { losses }\end{array}$ & $\begin{array}{l}\text { Requires access to internal } \\
\text { geometry of the machine } \\
\text { or the input-output relation } \\
\text { of the machine }\end{array}$ \\
& $\begin{array}{l}\text { Lower TPA at speed close } \\
\text { to base speed }\end{array}$ \\
\end{tabular}

\section{REFERENCES}

[1] G. Pellegrino, A. Vagati, P. Guglielmi and B. Boazzo, "Performance comparison between surface-mounted and interior PM motor drives for electric vehicle application," IEEE Trans. Ind. Electron., vol. 59, no. 2, pp. 803-811, Feb. 2012.

[2] J. Nerg, M. Rilla, V. Ruuskanen, J. Pyrhönen and S. Ruotsalainen, "Direct-driven interior magnet permanent-magnet synchronous motors for a full electric sports car," IEEE Trans. Ind. Electron., vol. 61, no. 8, pp. 4286-4294, Aug. 2014.

[3] J. Y. Alsawalhi and S. D. Sudhoff, "Design optimization of asymmetric salient permanent magnet synchronous machines," IEEE Trans. Energy Convers., vol. 31, no. 4, pp. 1315-1324, Dec. 2016.

[4] X. Zhu, Z. Xiang, L. Quan, Y. Chen and L. Mo, "Multimode optimization research on a multiport magnetic planetary gear permanent magnet machine for hybrid electric vehicles," IEEE Trans. Ind. Electron., vol. 65, no. 11 , pp. 9035-9046, Nov. 2018.

[5] A. Vagati, M. Pastorelli and G. Franceschini, "High-performance control of synchronous reluctance motors," IEEE Trans. Ind. Appl., vol. 33, no. 4, pp. 983-991, Jul.-Aug. 1997.

[6] F. Briz, M. W. Degner and R. D. Lorenz, "Analysis and design of current regulators using complex vectors," IEEE Trans. Ind. Appl., vol. 36, no. 3, pp. 817-825, May-Jun. 2000.

[7] H. Kim, M. W. Degner, J. M. Guerrero, F. Briz and R. D. Lorenz, "Discrete-Time Current Regulator Design for AC Machine Drives," in IEEE Trans. Ind. Appl., vol. 46, no. 4, pp. 1425-1435, Jul.-Aug. 2010.
[8] O. Wallmark, S. Lundberg and M. Bongiorno, "Input admittance expressions for field-oriented controlled salient PMSM drives," in IEEE Trans. Power Electron., vol. 27, no. 3, pp. 1514-1520, Mar. 2012.

[9] T. Lubin, H. Razik, and A. Rezzoug, "Magnetic saturation effects on the control of a synchronous reluctance machine," IEEE Trans. Energy Convers., vol. 17, no. 3, pp. 356-362, Sep. 2002.

[10] S. Yamamoto, T. Ara and K. Matsuse, "A method to calculate transient characteristics of synchronous reluctance motors considering iron loss and cross-magnetic saturation," in IEEE Trans. Ind. Appl., vol. 43, no. 1, pp. 47-56, Jan. 2007.

[11] Z. Qu, T. Tuovinen and M. Hinkkanen, "Inclusion of magnetic saturation in dynamic models of synchronous reluctance motors," 2012 XXth Int. Conf. Electric. Mach., Marseille, 2012, pp. 994-1000.

[12] T. S. Kwon, G. Y. Choi, M. S. Kwak, and S. K. Sul, "Novel flux weakening control of an IPMSM for quasi-six-step operation," IEEE Trans. Ind. Appl., vol. 44, no. 6, pp. 1722-1731, Nov. 2008.

[13] M. Meyer and J. Bocker, "Optimum control for interior permanent magnet synchronous motors (ipmsm) in constant torque and flux weakening range," 2006 12th Int. Power Elect. Motion Cont. Conf., Aug 2006, pp. $282-286$.

[14] H. W. de Kock, A. J. Rix and M. J. Kamper, "Optimal torque control of synchronous machines based on finite-element analysis," in IEEE Trans. Ind. Electron., vol. 57, no. 1, pp. 413-419, Jan. 2010.

[15] X. Chen, J. Wang, B. Sen, P. Lazari and T. Sun, "A high-fidelity and computationally efficient model for interior permanent-magnet machines considering the magnetic saturation, spatial harmonics, and iron loss effect," IEEE Trans. Ind. Electron., vol. 62, no. 7, pp. 4044-4055, July 2015.

[16] B. Mansouri and J. Piaton, "Magnetic saturation aids flux-weakening control: using lookup tables based on a static method of identification for nonlinear permanent-magnet synchronous motors," IEEE Electrif. Magazine, vol. 5, no. 4, pp. 53-61, Dec. 2017.

[17] M. H. Mohammadi and D. A. Lowther, "A computational study of efficiency map calculation for synchronous AC motor drives including cross-coupling and saturation effects," IEEE Trans. Magn., vol. 53, no. 6, pp. 1-4, June 2017.

[18] H. A. A. Awan, Z. Song, S. E. Saarakkala, and M. Hinkkanen, "Optimal torque control of saturated synchronous motors: Plug-and-play method," IEEE Trans. Ind. Appl., doi: 10.1109/TIA.2018.2862410.

[19] G. Y. Sizov, D. M. Ionel and N. A. O. Demerdash, "Modeling and parametric design of permanent-magnet AC machines using computationally efficient finite-element analysis," IEEE Trans. Ind. Electron., vol. 59, no. 6, pp. 2403-2413, June 2012.

[20] G. Luo, R. Zhang, Z. Chen, W. Tu, S. Zhang and R. Kennel, "A novel nonlinear modeling method for permanent-magnet synchronous motors," IEEE Trans. Ind. Electron., vol. 63, no. 10, pp. 6490-6498, Oct. 2016.

[21] M. F. Far, B. Mustafa, F. Martin, P. Rasilo and A. Belahcen, "FluxWeakening Control for IPMSM Employing Model Order Reduction," 2018 XIII Int. Conf. Electrical Machines (ICEM), Alexandroupoli, Greece, 2018, pp. 1510-1516.

[22] T. M. Jahns, G. B. Kliman and T. W. Neumann, "Interior PermanentMagnet Synchronous Motors for Adjustable-Speed Drives," in IEEE Trans. Ind. Appl., vol. IA-22, no. 4, pp. 738-747, Jul. 1986.

[23] M. Farzam Far, F. Martin, A. Belahcen, L. Montier and T. Henneron, "Orthogonal interpolation method for order reduction of a synchronous machine model," IEEE Trans. Magn., vol. 54, no. 2, pp. 1-6, Feb. 2018.

[24] M. Farzamfar, A. Belahcen, P. Rasilo, S. Clénet and A. Pierquin, "Model order reduction of electrical machines with multiple inputs," in IEEE Trans. Ind. Appl., vol. 53, no. 4, pp. 3355-3360, Jul.-Aug. 2017.

[25] G. Strang, Introduction to linear algebra, 4th ed., Wellesley, Massachusetts 02482: Wellesley - Cambridge Press, ch. 7, pp. 363-369, May 2009.

[26] G. Lei, J. Zhu, Y. Guo, Multidisciplinary design optimization methods for electrical machines and drive systems, 1st ed., power system series. Springer Berlin Heidelberg, ch. 2, pp. 51, Feb. 2016.

[27] S. Kim, Y. D. Yoon, S. K. Sul and K. Ide, "Maximum torque per ampere (MTPA) control of an IPM machine based on signal injection considering inductance saturation," IEEE Trans. Power Electron., vol. 28, no. 1, pp. 488-497, Jan. 2013.

[28] M. Farzamfar, A. Belahcen, P. Rasilo, S. Clénet and A. Pierquin, "Model order reduction of electrical machines with multiple inputs," in IEEE Trans. Ind. Appl., vol. 53, no. 4, pp. 3355-3360, Jul.-Aug. 2017.

[29] M. Farzam Far, F. Martin, A. Belahcen, L. Montier and T. Henneron, "Orthogonal interpolation method for order reduction of a synchronous machine model," in IEEE Trans. Magn., vol. 54, no. 2, pp. 1-6, Feb. 2018, 
[30] P. Rasilo, M. Lemesle, A. Belahcen, A. Arkkio and M. Hinkkanen, "Comparison of Finite-Element-Based State-Space Models for PM Synchronous Machines," in IEEE Trans. Energy Convers., vol. 29, no. 2, pp. 535-543, Jun. 2014

[31] M. Hinkkanen, H. Asad Ali Awan, Z. Qu, T. Tuovinen and F. Briz, "Current control for synchronous motor drives: direct discrete-time poleplacement design," in IEEE Trans. Ind. Appl., vol. 52, no. 2, pp. 15301541, Mar.-Apr. 2016.

[32] E. Armando, R. I. Bojoi, P. Guglielmi, G. Pellegrino and M. Pastorelli, "Experimental identification of the magnetic model of synchronous machines," in IEEE Trans. Ind. Appl., vol. 49, no. 5, pp. 2116-2125, Sept.Oct. 2013.

[33] L. Peretti, P. Sandulescu and G. Zanuso, "Self-commissioning of flux linkage curves of synchronous reluctance machines in quasi-standstill condition," in IET Electric Power Appl., vol. 9, no. 9, pp. 642-651, Nov. 2015

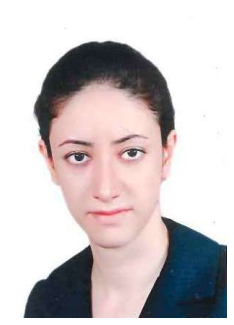

M. Farzam Far received her M.Sc. (Tech.) and D.Sc. (Tech.) degrees from Aalto University, Espoo, Finland in 2014 and 2019, respectively. She joined VTT Technical Research Centre of Finland in 2019, where she works as a Research Scientist in the Electrical powertrains and storage team. Her research interests include numerical modeling of electrical machines as well as mode order reduction and machine learning techniques.

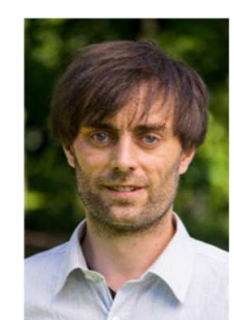

F. Martin received his engineer diploma of electrical engineering from Polytech'Nantes and his M.S. degree in electrical engineering from University of Nantes in 2009. He received the Ph.D. degree from the University of Nantes in 2013. He joined the department of electrical engineering and automation in Aalto University in 2014 where he works as a researcher in the group of computational electromechanics. His research topics include not only thermo-magnetomechanical constitutive model of magnetic materials, but also the model and optimization of electrical devices such as high-speed synchronous machine and reluctance machines.

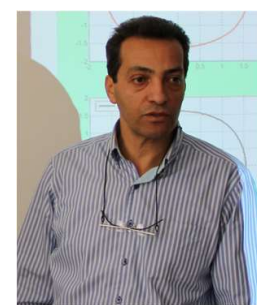

A. Belahcen (M13-SM15) received the M.Sc. (Tech.) and Doctor (Tech.) degrees from Helsinki University of Technology, Finland, in 1998 , and 2004, respectively. $\mathrm{He}$ is now Professor of electrical machines at Tallinn University of Technology, Estonia and Professor of Energy and Power at Aalto University, Finland. His research interest are numerical modeling of electrical machines, magnetic materials, coupled magneto-mechanical problems, magnetic forces, magnetostriction, and fault diagnostics.

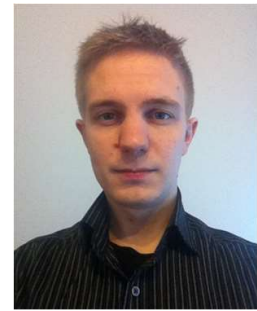

P. Rasilo received his M.Sc. (Tech.) and D.Sc. (Tech.) degrees from Helsinki University of Technology (currently Aalto University) and Aalto University, Espoo, Finland in 2008 and 2012 , respectively. He is currently working as an Assistant Professor at the Department of Electrical Engineering, Tampere University of Technology, Tampere, Finland. His research interests deal with numerical modeling of electrical machines as well as power losses and magnetomechanical effects in soft magnetic materials.

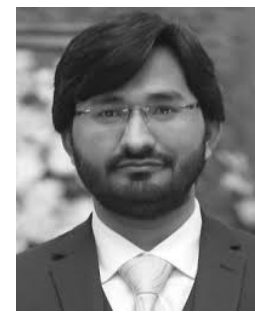

H. A. A. Awan received M.Sc. (Tech.) and D.Sc. (Tech.) degrees in electrical engineering from Aalto University, Espoo, Finland in 2015 and 2019 , respectively. He is currently working as a researcher at ABB Oy Drives, Helsinki, Finland. His main research interest include control of electric drives. He was the corecipient of the 2018 IEEE Industry Applications Society Industrial Drives Committee Best Paper Award. 